\title{
Exploring diagnostic performance of T2 mapping in diffuse glioma grading
}

\author{
Weibin Gu ${ }^{1 \#}$, Shiyuan Fang ${ }^{2 \#}$, Xinyi Hou ${ }^{1}$, Ding Ma ${ }^{1,3}$, Shaowu $\mathrm{Li}^{1,3,4}$ \\ ${ }^{1}$ Department of Radiology, Beijing Tiantan Hospital, Capital Medical University, Beijing, China; ${ }^{2}$ Department of Neurology, Peking Union Medical \\ College Hospital, Chinese Academy of Medical Sciences and Peking Union Medical College, Beijing, China; ${ }^{3}$ Beijing Neurosurgical Institute, \\ Capital Medical University, Beijing, China; ${ }^{4}$ National Clinical Research Center for Neurological Diseases, Beijing, China
}

\#These authors contributed equally to this work and are co-first authors.

Correspondence to: Shaowu Li, MD. Department of Radiology, Beijing Tiantan Hospital, Capital Medical University, Beijing 100050, China; Beijing Neurosurgical Institute, Capital Medical University, Beijing 100050, China; National Clinical Research Center for Neurological Diseases, Beijing, China. Email: lys5@sina.com.

Background: To evaluate the diagnostic performance of T2 mapping in differentiating WHO grade II glioma from high-grade glioma (HGG).

Methods: We conducted a single-center, retrospective diagnostic study. Confirmed diffuse glioma (WHO grade II-IV) patients who underwent post-contrast T1-weighted imaging, T2-weighted imaging, and T2 mapping were included. All diagnoses were based on histological and molecular tests. Seventy-five percent of cases were subsampled to generate receiver operating characteristic (ROC) curves and areas under the curve (AUC), while the remaining cases were used to test the accuracy of T2 mapping. Subsampling was repeated four times. Age, T2 relaxation time, and contrast-enhancement status were used to generate a multivariable ROC curve. T2 relaxation time was also used to generate ROC curves to predict the isocitrate dehydrogenase (IDH) status.

Results: A total of 159 patients were included in the study. After four repeats of subsampling, the AUCs of the T2 mapping ROC curve were 0.801 (95\% CI: 0.724-0.879), 0.795 (95\% CI: 0.714-0.875), 0.803 (95\% CI: $0.723-0.884$ ), and 0.801 (95\% CI: $0.716-0.886)$, with an average sensitivity of 0.753 and an average specificity of 0.767 . When applied to the remaining $25 \%$ of cases, the accuracy was $75 \%, 93.75 \%, 82.50 \%$, and $71.74 \%$. The AUC of the multivariable ROC was 0.927 (95\% CI: 0.882-0.971). IDH-mutant and IDH-wildtype gliomas have significantly different $\mathrm{T} 2$ relaxation times (146.28 and $124.10 \mathrm{~ms}$, respectively; $\mathrm{P}=0.001$ ), and the AUC of IDH-mutant prediction was 0.687 (95\% CI: 0.585-0.789).

Conclusions: Quantitative T2 mapping differentiated WHO grade II glioma from HGG with moderate sensitivity and specificity. Given the advantages of short acquisition times and the absence of a contrast agent, our study suggests the application of T2 mapping in pre-operative glioma grading is feasible.

Keywords: T2 mapping; diffuse glioma; grading; quantitative; magnetic resonance imaging (MRI)

Submitted Jul 27, 2020. Accepted for publication Mar 12, 2021.

doi: 10.21037/qims-20-916

View this article at: http://dx.doi.org/10.21037/qims-20-916

\footnotetext{
^ ORCID: 0000-0001-9889-9197.
} 


\section{Introduction}

Diffuse gliomas are the most prevalent primary central nervous system tumor in adults, with an annual incidence of 6 cases per 100,000 (1). The 2016 World Health Organization (WHO) Classification of central nervous system tumors categorizes glioma by molecular nature and histological characteristics (2). Based on histological examination, diffuse astrocytoma and oligodendroglioma are categorized as grade II gliomas, while anaplastic astrocytoma and anaplastic oligodendroglioma are categorized as grade III gliomas. Glioblastoma (GBM) accounts for the majority of grade IV gliomas. Grade II to IV gliomas are also referred to as diffuse gliomas due to their infiltrative nature into peritumoral brain tissues (3). Grade III and IV gliomas are collectively known as high-grade gliomas (HGGs), while grade I and II are known as low-grade gliomas (LGGs) (2). Glioma grading indicates different treatment responses and prognosis; For example, WHO grade IV glioma (mostly GBM) has a dismal 5 -year survival rate of $5 \%$ even with resection, radiotherapy, and temozolomide treatment (4).

Additionally, gliomas are also categorized according to their genetic makeup. Based on the isocitrate dehydrogenase (IDH) gene, WHO grade III gliomas are categorized as IDH-mutant (mt) or IDH-wildtype (wt) glioma, whereby IDH-mt gliomas have a more favorable prognosis than their IDH-wt counterparts (3). Therefore, pre-surgical prediction of glioma grading and molecular status has become clinically important.

Despite the crucial role that pre-operative tumor evaluation plays in treatment decision-making, current grading methods involve post-operative histological and molecular testing. It is reported that certain imaging features are associated with glioma's histological features, molecular subtypes, and grading $(3,4)$. HGGs are usually contrast-enhanced, while the sensitivity of gadoliniumenhanced T1-weighted image (T1G) for differentiating high- and low-grade oligodendroglioma is only $63 \%$; enhancement is also not usually seen in anaplastic astrocytoma (3). Dynamic susceptibility perfusion magnetic resonance imaging (MRI) was reported to have high accuracy [area under the curve (AUC) 0.86] in differentiating high- and low-grade astrocytoma, while for oligodendroglioma, the AUC drops to 0.61 (5). Arterial spin labeling (ASL) MRI had an improved accuracy (AUC 0.90) in differentiating HGGs from LGGs (6); however, ASL has a low signal-to-noise ratio which requires multiple image-pairs to overcome (7). Texture analysis derived from T1-weighted imaging (T1WI), T2-weight imaging (T2WI), T1G, and T2-fluid attenuated inversion recovery (FLAIR) had an AUC of 0.86 in differentiating grade II from grade III gliomas (8). O- $\left(2-\left[{ }^{18} \mathrm{~F}\right]\right.$-fluoroethyl)-Ltyrosine positron emission tomography $\left({ }^{18} \mathrm{~F}-\mathrm{FET} \mathrm{PET}\right)$ shows an AUC of 0.80-0.83 in differentiating LGGs from HGGs (9); however, this requires injection of radioactive agents and has potential hazards, especially for patients who need repetitive scanning. Currently, there is no reliable, quantitative, non-invasive imaging method that does not involve injecting agents.

Quantitative T2 measurements reflect water accumulation, blood volume, myelin loss, and gliosis $(10,11)$. In the past decade, quantitative $\mathrm{T} 2$ values have been utilized in glioma in many aspects. In 2010, the Response Assessment in NeuroOncology (RANO) working group applied the T2/FLAIR sequences to glioma imaging features (12), highlighting the importance of $\mathrm{T} 2$ relaxation time in glioma evaluation. In 2012, Ellingson et al. adopted differential quantitative T2 relaxometry mapping to quantify edema reduction after bevacizumab treatment (13). In 2013, Hattingen et al. reported that T2 mapping could monitor non-enhancing tumor progression (14). In 2020, Kern et al. used T2 mapping to predict the IDH status in grade II/III gliomas (15).

Quantitative T2 measurements have traditionally required long acquisition times; however, recent advances by Siemens Healthcare (Erlangen, Germany) have combined the MARTINI (Model-based Accelerated Relaxometry by Iterative Non-linear Inversion) approach and the GRAPPA (Generalized Auto-calibrating Partially Parallel Acquisition) reconstruction to generate GRAPPATINI, which achieves high-resolution T2 mapping of the whole brain within two minutes (10). Our study used this GRAPPATINI approach from Siemens Healthcare to quantify T2 relaxation times in gliomas. Our study aimed to explore this T2 mapping approach's diagnostic performance in differentiating WHO grade II gliomas from HGGs.

\section{Methods}

\section{Participants}

We conducted a single-center, retrospective diagnostic study. Data from consecutive patients with suspected glioma admitted to Beijing Tiantan Hospital from February 2016 to September 2018 were recruited for the study. Inclusion criteria included: (I) supratentorial, parenchymal, confirmed diffuse glioma (WHO grade II-IV); (II) availability of 
histopathology results; (III) availability of molecular and/ or immunohistochemistry test results (DNA sequencing and immunohistochemistry for IDH mutations, ATRX loss, and MGMT methylation; fluorescence in situ hybridization for $1 \mathrm{p} / 19 \mathrm{q}$ codeletion); (IV) T2 mapping and standard MRI (including T1G and T2WI) results available before resection or therapy. The Institutional Review Board of Beijing Tiantan Hospital, Capital Medical University approved the study, and all participants provided written informed consent.

\section{Design}

The research was reported following the STARD 2015 guidelines for reporting diagnostic accuracy studies (16). The index test was T2 mapping, and the quantitative T2 value of each tumor's solid region was used to generate receiver operator characteristic (ROC) curves. The coordinate with the highest Youden index was used as the cut-off point. Tumors whose T2 values were higher than the cut-off point were diagnosed as WHO grade II glioma, and tumors with a T2 value lower than the cut-off point were diagnosed as HGG. The reference test integrates pathological tests and molecular tests, strictly following the 2016 WHO classification of tumors of the central nervous system (2). Inconclusive pathological and molecular tests were classified as glioma not otherwise specified (NOS).

We randomly selected $75 \%$ of the total cases to generate a ROC curve and identified a cut-off point. We then tested the accuracy of this cut-off point using the remaining $25 \%$ of cases. We repeated this procedure four times and consequently constructed four ROC curves and calculated four AUCs. We then calculated the mean cut-off value and applied this to all 159 participants to calculate the AUC, accuracy, sensitivity, and specificity.

To fully evaluate the value of T2 mapping in combination with T1G MRI, we further included age, gender, T2 relaxation time, and enhancement status to fit a multivariable logistic regression model. This model was used to generate a multivariable ROC curve and ascertain its AUC. To evaluate enhancement status contribution, another AUC of the ROC curve was calculated from the enhancement status diagnostic tool data only.

\section{Data acquisition}

We used the Siemens Healthcare GRAPPATINI T2 mapping software prototype (10). All acquisitions were performed under 3 Tesla (MAGNETOM Prisma; Siemens Healthcare Erlangen, Germany) scanners using commercially available 20-channel, 32-channel, and 64-channel head coils. T1G, T2WI, and T2 mapping sequences were performed using relevant parameters (Table 1). T1G imaging was acquired after intravenous gadobutrol diethylenetriaminepentaacetic acid (DTPA) injection with a dosage of $0.1 \mathrm{mmol} / \mathrm{kg}$.

\section{Imaging analysis}

Two blinded experienced neuroradiologists (each with more than ten years of practicing) labeled and measured each tumor's region of interest (ROI) using a postprocessing workstation (Syngo, Siemens Healthcare). The solid tumor region was defined as the enhanced region for enhanced tumors, excluding vessels and choroid plexus. For unenhanced tumors, the solid tumor region was defined as the hyperintense area on T2WI, which appeared less bright than the cerebrospinal fluid and more heterogeneous than edema. Cysts, necrosis, hemorrhages, and calcifications on other pre-surgical routine images-including plain CT scan, T1WI, T2WI, FLAIR, and DWI (14)-were excluded. Three ROIs with an area of $0.03 \mathrm{~cm}^{2}$ each were randomly selected from each tumor region and measured for T2 values; the mean value was used as the T2 value of the solid tumor region.

\section{Statistics}

All statistical analyses were performed with SPSS Version 23 (International Business Machines Corporation). Descriptive results were presented as percentages or medians with interquartile range (IQR). A Shapiro-Wilk test was used to test for normal distribution of continuous data. A Mann-Whitney $U$ test was used to compare the T2 relaxation times between gliomas of different classifications. A multivariable logistic regression model was fitted using the stepwise forward method. The diagnostic performance of T2 mapping and T2 mapping plus other variables was assessed using ROC curves and AUCs. Statistical significance was defined as $\mathrm{P}<0.05$.

\section{Results}

\section{Clinical characteristics}

Initial eligibility screening identified 234 patients with suspected glioma. There were 66 patients excluded due to 
Table 1 MRI acquisition parameters

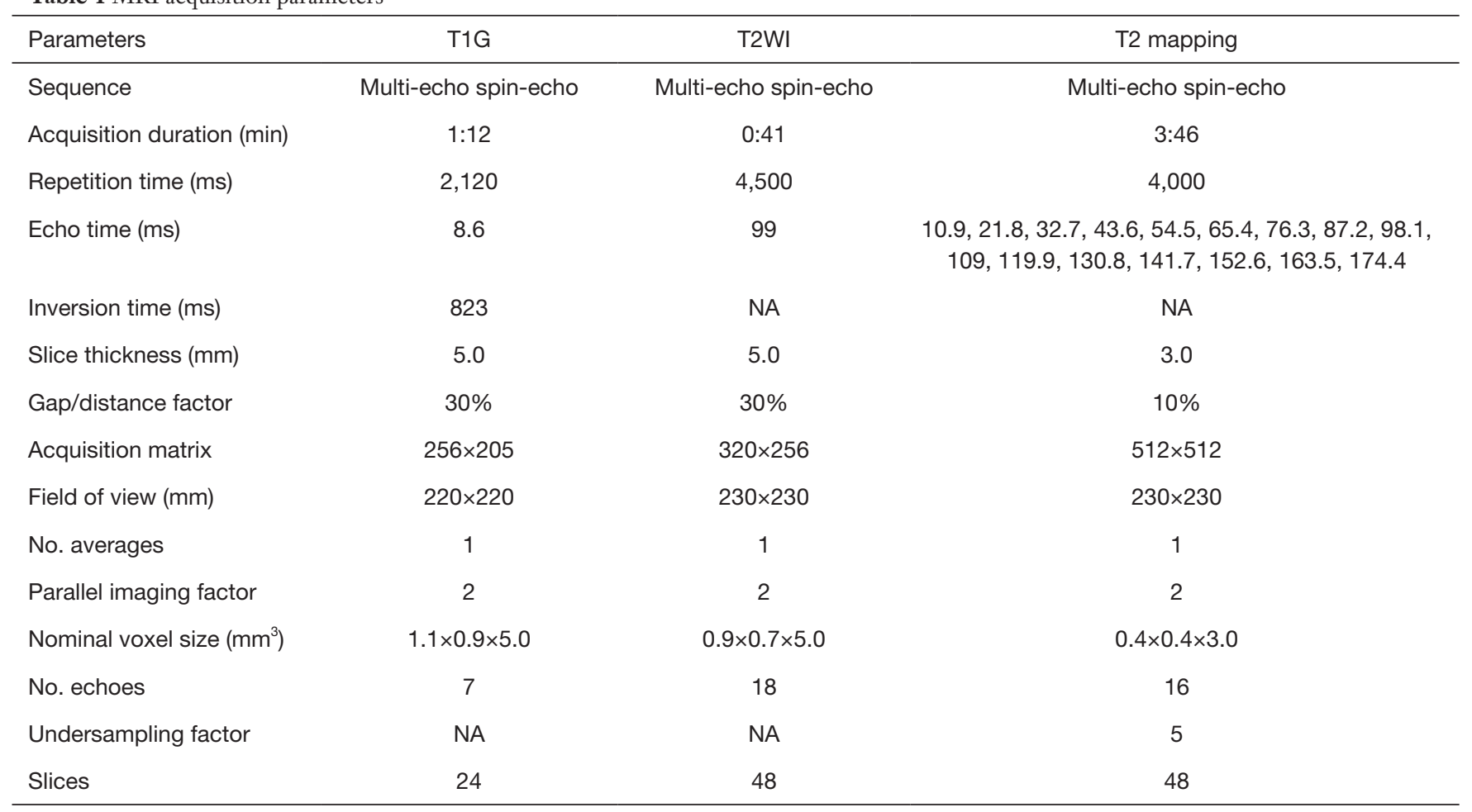

T1G, T1 imaging after gadolinium injection; NA, not applicable.

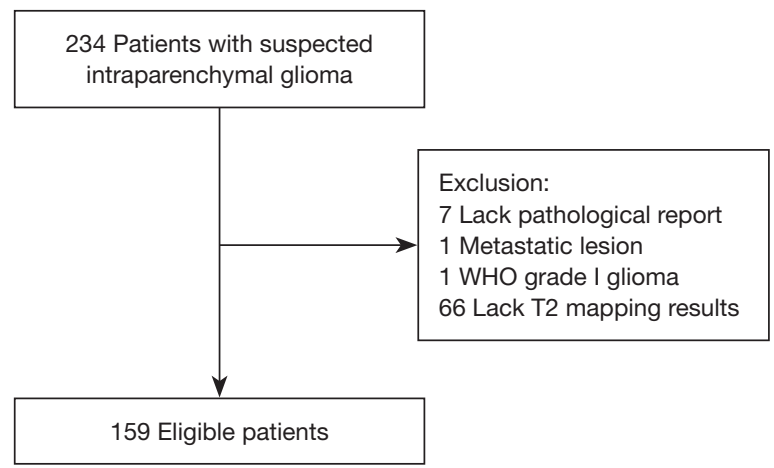

Figure 1 Flow diagram of patient inclusion.

lack of T2 mapping results and a further 7 excluded for the absence of a post-operative pathological report. One patient with lung cancer metastases and one patient with pilocytic astrocytoma were also excluded. A total of 159 patients with confirmed diffuse gliomas were recruited for the study. Figure 1 is a summary flow diagram of patient selection. The interval between radiological imaging and surgery was less than one week.

Study participant characteristics are summarized in
Table 2. Participants' median age was 46 years old (IQR, $35-53$ years old), and 81 (50.9\%) participants were female. There were 73 (45.9\%) participants diagnosed with WHO grade II glioma, 40 (25.2\%) with WHO grade III glioma, and 46 (28.9\%) with WHO grade IV glioma. Seventy six $(47.8 \%)$ participants demonstrated enhancing tumors on T1G MRI, while 83 (52.2\%) were unenhanced. The most common tumor classification was diffuse astrocytoma, IDH$\mathrm{mt}$ (22 cases, $13.8 \%$ ), followed by GBM, IDH-mt, and oligodendroglioma, IDH-mt, and 1p/19q-codeleted (both 21 cases, $13.2 \%)$.

\section{T2 relaxation times}

Figure 2 shows examples of T2WI images, T1G images, and the corresponding pseudo-color T2 maps. The median T2 relaxation times of grade II glioma and HGGs were 164.43 (IQR, 147.99, 180.87) $\mathrm{ms}$ and 126.75 (IQR, 96.93, 156.57) $\mathrm{ms}$, respectively. The median value of grade II glioma was significantly higher than that of HGGs $(\mathrm{P}<0.001)$.

Table 3 and Figure 3 show the median T2 relaxation times and comparisons between molecular categories. The $\mathrm{T} 2$ relaxation time distribution was significantly different 
Table 2 Clinical characteristics of participants

\begin{tabular}{|c|c|}
\hline Characteristic & No. of patients (\%) \\
\hline \multicolumn{2}{|l|}{ Gender } \\
\hline Male & $78(49.1)$ \\
\hline Female & $81(50.9)$ \\
\hline Age (years, median, IQR) & $46(35,53)$ \\
\hline II & $73(45.9)$ \\
\hline III & $40(25.2)$ \\
\hline IV & $46(28.9)$ \\
\hline \multicolumn{2}{|l|}{ Contrast enhancement status } \\
\hline \multicolumn{2}{|l|}{ Classification } \\
\hline Diffuse astrocytoma, IDH-mutant & $22(13.8)$ \\
\hline Diffuse astrocytoma, IDH-wildtype & $3(1.9)$ \\
\hline Diffuse astrocytoma, NOS & $8(5.0)$ \\
\hline Anaplastic astrocytoma, IDH-mutant & $9(5.7)$ \\
\hline Anaplastic astrocytoma, IDH-wildtype & $7(4.4)$ \\
\hline Anaplastic astrocytoma, NOS & $1(0.6)$ \\
\hline Glioblastoma, IDH-wildtype & $14(8.8)$ \\
\hline Anaplastic oligodendroglioma, NOS & $6(3.8)$ \\
\hline Oligoastrocytoma, NOS & $13(8.2)$ \\
\hline Anaplastic oligoastrocytoma, NOS & $7(4.4)$ \\
\hline Pleomorphic xanthoastrocytoma (II) & $1(0.6)$ \\
\hline Pilomyxoid astrocytoma (II) & $1(0.6)$ \\
\hline Diffuse midline glioma (IV) & $1(0.6)$ \\
\hline Gliosarcoma (IV) & $1(0.6)$ \\
\hline
\end{tabular}

IQR, interquartile range; WHO, World Health Organization; IDH, isocitrate dehydrogenase; NOS, not otherwise specified.

between IDH-mt and IDH-wt gliomas $(\mathrm{P}=0.001)$ and between MGMT-methylated and MGMT-unmethylated gliomas $(\mathrm{P}=0.03)$. Borderline significance was found between $1 \mathrm{p} / 19 \mathrm{q}$-codeleted and not co-deleted gliomas $(\mathrm{P}=0.05)$. No distribution difference was observed between ATRX gene loss and present gliomas; however, when 

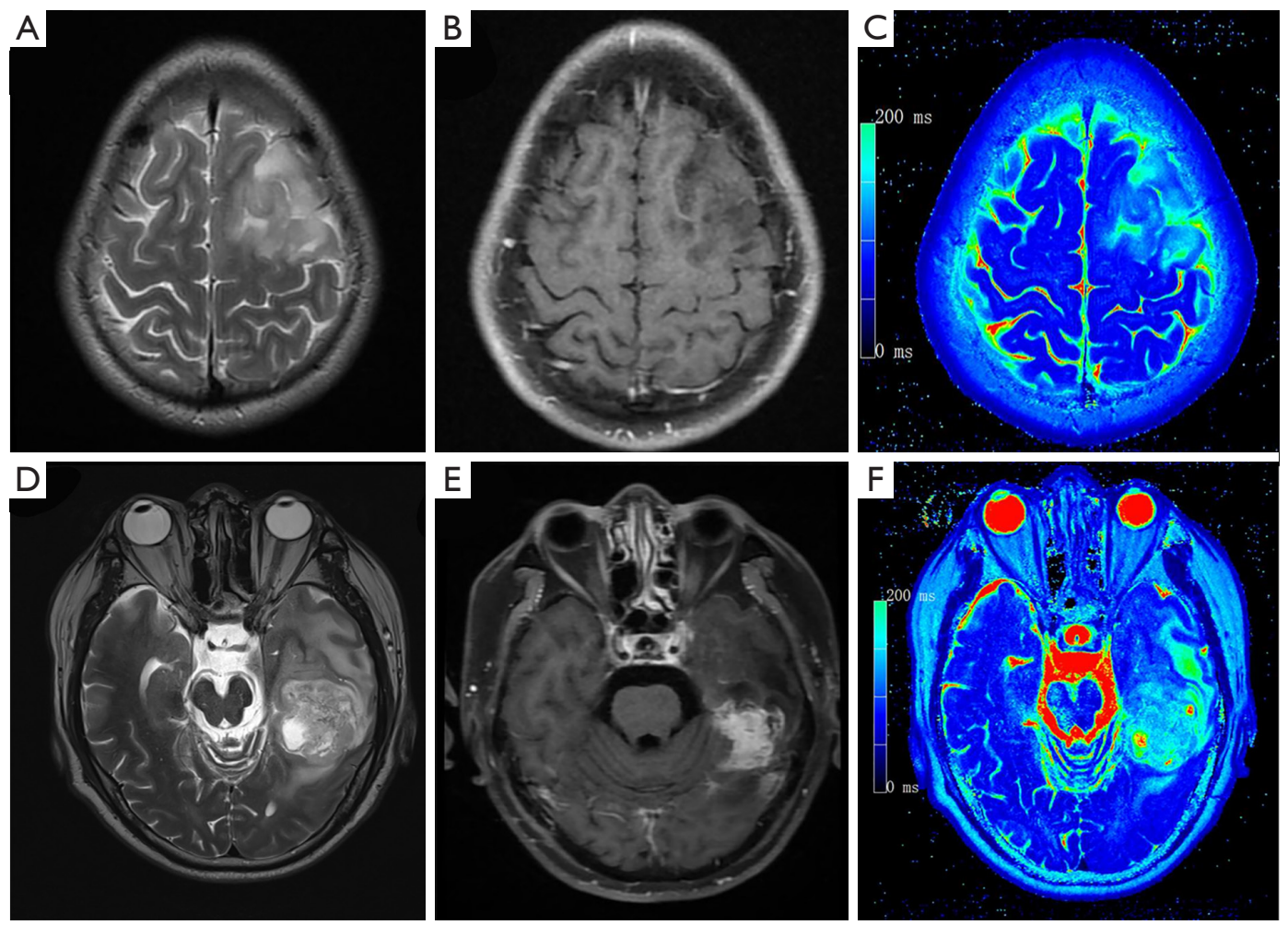

Figure 2 MRI examples of WHO II and III gliomas. First column: T2WI; Second column: T1 contrast enhanced imaging; Third column: T2 mapping pseudo-color photo. First line: a 31-year-old male with a left frontal-parietal astrocytoma (WHO II). Second line: a 49-year-old male with a left temporal glioblastoma (WHO IV).

Table 3 T2 relaxation times of different glioma classifications

\begin{tabular}{lccc}
\hline Molecular status & $\begin{array}{c}\text { No. of patients } \\
(\%)\end{array}$ & $\begin{array}{c}\text { T2 relaxation } \\
\text { times (median) }\end{array}$ & P value \\
\hline IDH & 87 & 146.28 & 0.001 \\
IDH-mt & 41 & 124.10 & \\
IDH-wt & & & \\
1p/19q-codeletion & 40 & 147.05 & 0.05 \\
Co-deleted & 58 & 135.36 & \\
Not co-deleted & & & \\
ATRX & 36 & 141.86 & 0.591 \\
ATRX loss & 60 & 142.42 & \\
ATRX present & & & \\
MGMT & 114 & 142.57 & 0.031 \\
Methylated & 21 & 123.81 & \\
Unmethylated & &
\end{tabular}

T2 relaxation times were used as a diagnostic tool to predict molecular category, they could not make accurate predictions, with all AUCs were between 0.5-0.7. Table 4 shows the ROC analysis for predicting the IDH status, with an AUC of 0.687 .

\section{ROC for diagnosing $W H O$ grade II glioma from $H G G$}

In WHO grade II glioma, the T2 relaxation time of the solid tumor region was higher than that of HGG. The mean cut-off point between WHO grade II glioma and HGG was 141.48 ms. Figure 4 shows ROC curves of T2 mapping, each built with the randomly selected $75 \%$ of cases. The AUC of these curves were $0.801,0.795,0.803$, and 0.801 . The cut-off points from these curves were then used to test the accuracy of T2 mapping on the remaining $25 \%$ of the cases; the accuracy was $21 / 28$ (75\%), 30/32 (93.75\%), 33/40 $(82.50 \%)$, and $33 / 46(71.74 \%)$. Table 4 summarizes the main 

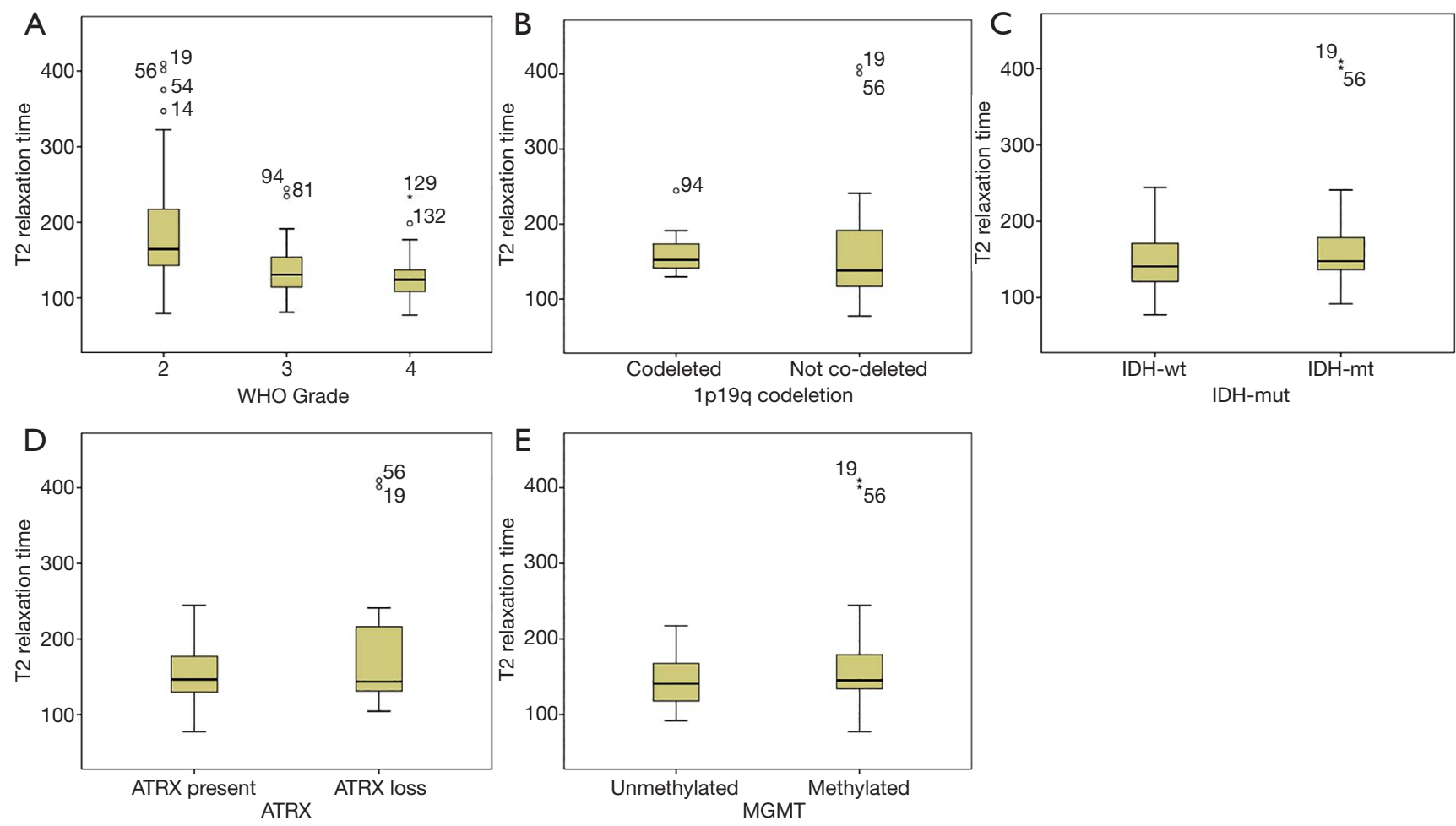

Figure 3 T2 relaxation times of different glioma classifications. (A) The different T2 relaxation times of WHO grade II, III and IV gliomas; $(\mathrm{B}, \mathrm{C}, \mathrm{D}, \mathrm{E})$ the $\mathrm{T} 2$ relaxation time distribution between gliomas of different genetic status. *, Outliers.

Table 4 Summary of all ROC curves

\begin{tabular}{|c|c|c|c|c|c|c|}
\hline Variables & AUC & $95 \% \mathrm{Cl}$ & Cut-off point & Accuracy (\%) & Sensitivity & Specificity \\
\hline \multirow{3}{*}{$\begin{array}{l}\text { T2 mapping } \\
\text { (subsampling } 75 \% \text { of patients) }\end{array}$} & 0.795 & $(0.714,0.875)$ & 141.35 & 30/32 (93.75) & 0.750 & 0.746 \\
\hline & 0.803 & $(0.723,0.884)$ & 142.75 & $33 / 40(82.50)$ & 0.755 & 0.754 \\
\hline & 0.801 & $(0.716,0.886)$ & 139.11 & $33 / 46(71.74)$ & 0.774 & 0.763 \\
\hline T2 mapping (all patients) & 0.803 & $(0.733,0.873)$ & 142.57 & $121 / 159(76.10)$ & 0.753 & 0.767 \\
\hline T2 mapping, $\mathrm{T} 1 \mathrm{G}$, and age & 0.927 & $(0.882,0.971)$ & NA & NA & 0.877 & 0.919 \\
\hline $\mathrm{T} 1 \mathrm{G}$ & 0.869 & $(0.808,0.929)$ & NA & NA & 0.877 & 0.860 \\
\hline T2 mapping (predicting IDH status) & 0.687 & $(0.585,0.789)$ & 131.45 & $89 / 128(69.53)$ & 0.713 & 0.659 \\
\hline
\end{tabular}

T1G, T1-weighted gadolinium-enhanced; IDH, isocitrate dehydrogenase; NA, not applicable.

findings of the ROC curves.

\section{Multivariable ROC}

The multivariate binary logistic regression model included variables of age, enhancement status, and $\mathrm{T} 2$ relaxation.
Gender was not included in the multivariate model as it did not show statistical significance in the univariate regression model. The AUC of the multivariable ROC curve was 0.927 (95\% CI: 0.882-0.971). For diagnosis based on enhancement status, the AUC was 0.869 (95\% CI: $0.808-$ 0.929), which was not significantly higher than the AUC 
A

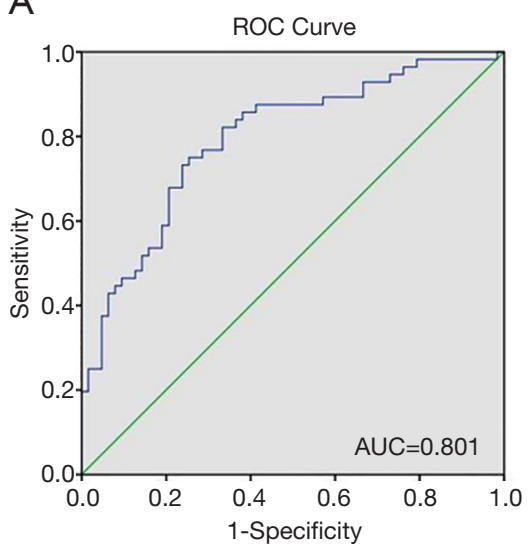

D

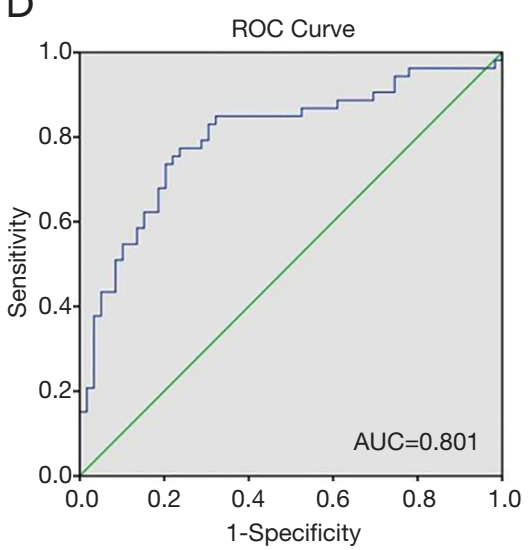

G

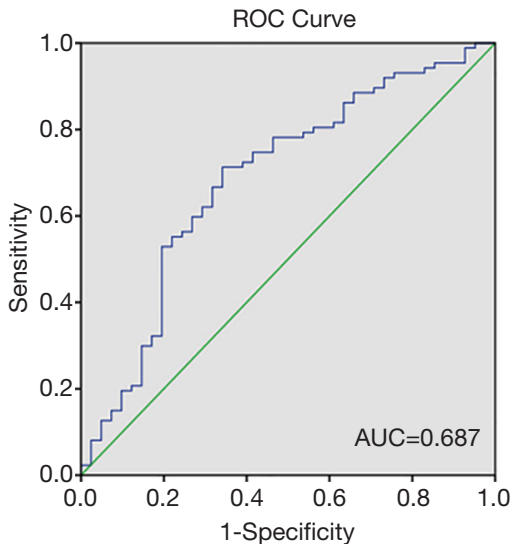

B

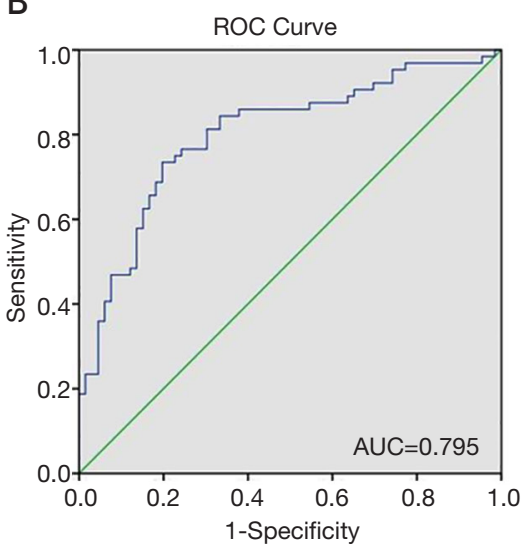

E

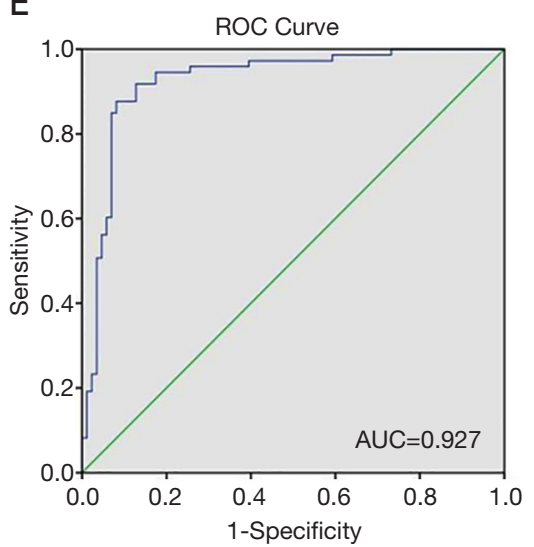

C

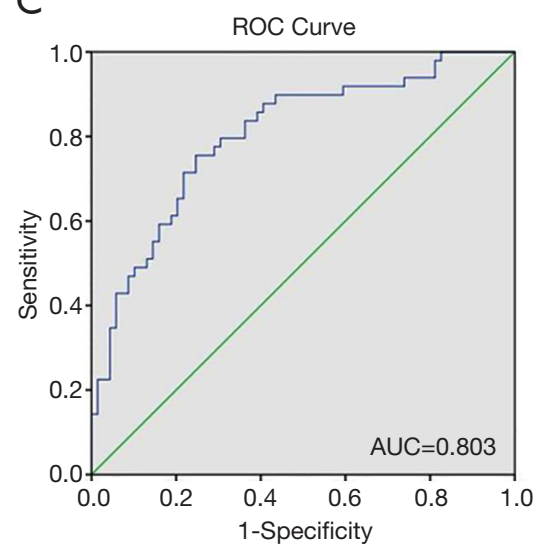

$\mathrm{F}$

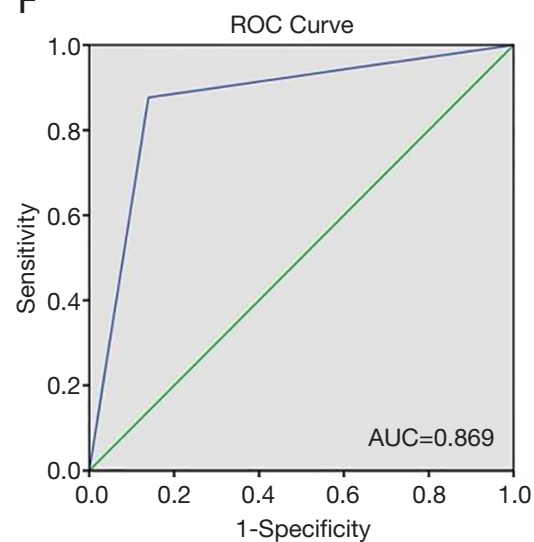

Figure 4 Univariable ROC curves of T2 relaxation times and multivariate ROC curves in diagnosing grade II glioma from HGGs and predicting IDH status. Panel A through D are generated by using single T2 mapping as the diagnostic tool, and each panel represents one subsampling. Panel $\mathrm{E}$ is a multivariate ROC curve using age, T2 mapping and contrast enhancement status. Panel $\mathrm{F}$ is derived from single contrast enhancement status. Panel G is shows T2 mapping used to predict IDH mutation. ROC, receiver operating characteristic; HGG, high-grade glioma; IDH, isocitrate dehydrogenase. 
for diagnosis based on T2 mapping. Figure 4 shows the multivariable ROC curve and the ROC curve for diagnosis based on enhancement status.

\section{Discussion}

We conducted a single-center, retrospective diagnostic study to explore the diagnostic performance of T2 mapping in differentiating WHO grade II glioma from HGG. T2 mapping appeared to be an appropriate diagnostic tool (AUC, 0.803; sensitivity, 0.753; specificity, 0.767), especially when used combined with patient age and glioma enhancement status data (AUC, 0.927; sensitivity, 0.877; specificity, 0.919). Study data showed that gliomas with short T2 relaxation times were likely to be HGGs, and gliomas with long $\mathrm{T} 2$ relaxation times were likely to be WHO grade II gliomas. Median T2 relaxation time was also significantly different between different molecular categories; specifically, IDH-mt gliomas had longer T2 relaxation times than IDH-wt gliomas. T2 mapping is noninvasive and requires no contrast agent, making it a viable prospect for pre-operative diffuse glioma grading.

WHO glioma grades are strongly associated with various treatment responses and prognoses. The study results showed that HGGs and LGGs have distinct T2 relaxation times, indicating that $\mathrm{T} 2$ mapping can provide diagnostic, therapeutic, and prognostic information. Maximum resection is recommended where possible for all glioma patients, while the first-line treatment for diffuse astrocytic and oligodendroglial tumors is either watch-and-wait or radiotherapy, followed by procarbazine, lomustine, and vincristine chemotherapy (PCV). In contrast, the first-line treatment for anaplastic astrocytoma and oligodendroglioma (WHO grade III) is radiotherapy and PCV (1). The use of T2 mapping to predict WHO grading before biopsy or surgery could assist in making clinical treatment choices.

Our study indicates that IDH-mt gliomas have longer T2 relaxation times than IDH-wt gliomas; this is also reflected in results published by Kern et al., which compared the $\mathrm{T} 2$ relaxation times between IDH-wt and IDH-mt gliomas using a similar T2 mapping algorithm (using 12 different echo times, 13.8-165.6 ms) (15). However, our ROC curve suggests that the feasibility of using T2 relaxation times to predict the IDH status is suboptimal. Additionally, we found borderline significance $(\mathrm{P}=0.05)$ of $\mathrm{T} 2$ relaxation times between $1 \mathrm{p} / 19 \mathrm{q}$ codeleted gliomas and 1p/19q not co-deleted gliomas; therefore, T2 mapping may be capable of differentiating glioma molecular subtypes.
Abundant literature has shown that the molecular subtypes are important biomarkers for prognosis and in treatment decision making. The existence of either IDH-mt or $1 \mathrm{p} / 19 \mathrm{q}$ codeletion is associated with favorable outcomes: WHO grade II, IDH-mt, and $1 \mathrm{p} / 19 \mathrm{q}$-codeleted gliomas have a median overall survival of 9.7 years; WHO grade II, IDH$\mathrm{mt}$, and non-codeleted gliomas have an overall survival of 3.6 years; and WHO grade II, IDH-wt and non-codeleted gliomas have an overall survival of 0.6 years (17). Similar patterns have also been seen in WHO grade III gliomas. Regarding treatment response, IDH-mt and $1 \mathrm{p} / 19 \mathrm{q}$ codeletion are also associated with preferable responses to radiotherapy. The median overall survival of IDH-mt or $1 \mathrm{p} / 19 \mathrm{q}$-coledeted gliomas under radiotherapy is 5.7 and 7.3 years respectively; however, the median overall survival of IDH-wt or $1 \mathrm{p} / 19 \mathrm{q}$ non-codeleted gliomas under the same treatment is only 1.8 and 2.7 years, respectively (17). Assessing glioma molecular profile would therefore assist with patient communication and decision making.

2016 WHO classification update introduced the concept of a 'layered diagnosis' for gliomas. This terminology suggests that the full diagnosis of a glioma (layer 1) should include its histologic classification (layer 2), WHO grade (layer 3), and molecular information (layer 4) (18). Our data suggest that T2 mapping could be used to predict glioma WHO grade, possibly including molecular information, based on the layered diagnosis model.

T2 mapping has certain strengths over other imaging modalities. Cuccarini et al. reported that perfusion-weighted imaging, diffusion-weighted imaging, and magnetic resonance spectroscopy each diagnosed LGGs with the sensitivity of $0.69,0.91,0.64$, and specificity of $0.54,0.46$, and 0.58 , respectively (19); however, this research enrolled patients with suspected LGGs, introducing major selection bias. Falk Delgado et al. conducted a meta-analysis of the diagnostic performance of ASL MRI between HGGs and LGGs (6); in the study of 505 patients, the AUC of the maximum of the regional cerebral blood flow reached 0.90 . While ASL MRI offers a noninvasive alternative that requires no contrastagent injection, the meta-analysis included studies that did not follow the updated 2016 WHO classification system and had heterogenous ROI placement. Verger et al. reported the AUC for HGG and LGG diagnosis using ${ }^{18} \mathrm{~F}-\mathrm{FET}$ PET was $0.80-0.83$ (9), which was slightly higher than T2 mapping in our study; however, oligodendroglioma-especially low-grade oligodendroglioma, which has a high uptake of ${ }^{18} \mathrm{~F}$-FET - were underrepresented in this study.

Regarding other novel technologies, Jeong et al. adopted 
machine-learning to classify GBM from LGG by extracting delta-radiomic features from dynamic susceptibility contrastenhanced MRI (20). The analysis showed that this model could consistently predict tumor grade with an AUC of 0.94; while the results are promising, this study had a small 25 patient sample size whose tumor gradings were based on 2007 WHO classification. Various imaging techniques are being explored for HGG and LGG diagnosis, with perfusion MRI being the most promising one.

We found the combination of T2 mapping and T1G elevated the AUC to 0.927 , higher than T2 mapping or T1G alone. While contrast enhancement is usually a sign of HGGs, this mostly applies to GBMs (3) as the newly generated vessels within GBMs have poor blood-brain barrier, causing leakage of contrast agents.

Regarding the utility of imaging techniques, T1G only provides qualitative, binary results compared with the quantitative results seen in T2 mapping. This quantitative nature of T2 mapping allows physicians to better monitor disease progression and treatment response and compare imaging data from different medical centers. Additionally, $\mathrm{T} 1 \mathrm{G}$ is not possible in all patients, as some patients are allergic to the contrast agent. Overall, T2 mapping and T1G's combination improved the diagnostic performance of differentiating grade II and grade III gliomas.

Our study has several strengths. Firstly, our study's newly-developed GRAPPATINI package has high reproducibility and low inter-observer variance (10). Secondly, our reference test was following the updated 2016 WHO glioma classification, using both pathological exams and genetic and/or immunohistochemistry. Thirdly, the single-center study's large sample size allowed four repetitions of patient subsampling, providing strong internal validation of results.

The study also had several limitations. Firstly, our study used retrospective analysis, and we suggest that the true potential of T2 mapping should be further examined under prospective designs. Secondly, the potential of T2 mapping to predict tumor infiltration and disease progression was not explored, as this requires local biopsies to validate. Thirdly, our study is a single-center experience; given the robust quantification among different scanners is a proposed advantage of the T2 mapping system, external validation in a multi-site study would better establish its diagnostic performance.

Future studies should explore two further aspects of T2 mapping diagnosis. Firstly, external validation is needed to verify the quantitative properties of $\mathrm{T} 2$ mapping and ascertain its glioma grading performance across multiple sites. Secondly, since non-enhancing lesion progression on T2 and/or FLAIR images now factors into response assessment, , future research using prognostic data could explore the role of $\mathrm{T} 2$ mapping in monitoring lesion expansion, high-grade transition, and recurrence after resection, with the possibility of detecting the lesion at an earlier stage than T1G.

T2 mapping is suitable for differentiating WHO grade II gliomas from HGGs. When combined with T1G, it has excellent accuracy. Gliomas of different IDH and/ or $1 \mathrm{p} / 19 \mathrm{q}$-codeletion statuses have different $\mathrm{T} 2$ mapping values. T2 mapping could have clinical applications for pre-operative glioma grading and molecular subtype predictions.

\section{Acknowledgments}

Funding: None.

\section{Footnote}

Conflicts of Interest: All authors have completed the ICMJE uniform disclosure form (available at http://dx.doi. org/10.21037/qims-20-916). The authors have no conflicts of interest to declare.

Ethical Statement: The Institutional Review Board of Beijing Tiantan Hospital Affiliated to Capital Medical University approved this study. All participants provided written informed consent.

Open Access Statement: This is an Open Access article distributed in accordance with the Creative Commons Attribution-NonCommercial-NoDerivs 4.0 International License (CC BY-NC-ND 4.0), which permits the noncommercial replication and distribution of the article with the strict proviso that no changes or edits are made and the original work is properly cited (including links to both the formal publication through the relevant DOI and the license). See: https://creativecommons.org/licenses/by-nc-nd/4.0/.

\section{References}

1. Weller M, van den Bent M, Tonn JC, Stupp R, Preusser M, Cohen-Jonathan-Moyal E, Henriksson R, Le Rhun E, Balana C, Chinot O, Bendszus M, Reijneveld JC, Dhermain F, French P, Marosi C, Watts C, Oberg I, 
Pilkington G, Baumert BG, Taphoorn MJB, Hegi M, Westphal M, Reifenberger G, Soffietti R, Wick W. European Association for Neuro-Oncology (EANO) guideline on the diagnosis and treatment of adult astrocytic and oligodendroglial gliomas. Lancet Oncol 2017;18:e315-e329.

2. Louis DN, Perry A, Reifenberger G, von Deimling A, Figarella-Branger D, Cavenee WK, Ohgaki H, Wiestler OD, Kleihues P, Ellison DWJAN. The 2016 World Health Organization Classification of Tumors of the Central Nervous System: a summary. Acta neuropathol 2016;131:803-20.

3. van den Bent MJ, Smits M, Kros JM, Chang SM. Diffuse Infiltrating Oligodendroglioma and Astrocytoma. J Clin Oncol 2017;35:2394-401.

4. Alexander BM, Cloughesy TF. Adult Glioblastoma. J Clin Oncol 2017;35:2402-9.

5. Delgado AF, Delgado AF. Discrimination between Glioma Grades II and III Using Dynamic Susceptibility Perfusion MRI: A Meta-Analysis. AJNR Am J Neuroradiol 2017;38:1348-55.

6. Falk Delgado A, De Luca F, van Westen D, Falk Delgado A. Arterial spin labeling MR imaging for differentiation between high- and low-grade glioma-a meta-analysis. Neuro Oncol 2018;20:1450-61.

7. Spann SM, Kazimierski KS, Aigner CS, Kraiger M, Bredies K, Stollberger R. Spatio-temporal TGV denoising for ASL perfusion imaging. Neuroimage 2017;157:81-96.

8. Zhou H, Vallières M, Bai HX, Su C, Tang H, Oldridge D, Zhang Z, Xiao B, Liao W, Tao Y, Zhou J, Zhang P, Yang L. MRI features predict survival and molecular markers in diffuse lower-grade gliomas. Neuro Oncol 2017;19:862-70.

9. Verger A, Filss CP, Lohmann P, Stoffels G, Sabel M, Wittsack HJ, Kops ER, Galldiks N, Fink GR, Shah NJ, Langen KJ. Comparison of (18)F-FET PET and perfusion-weighted MRI for glioma grading: a hybrid PET/MR study. Eur J Nucl Med Mol Imaging 2017;44:2257-65.

10. Hilbert T, Sumpf TJ, Weiland E, Frahm J, Thiran JP, Meuli R, Kober T, Krueger G. Accelerated T(2) mapping combining parallel MRI and model-based reconstruction: GRAPPATINI. J Magn Reson Imaging 2018;48:359-68.

11. Oh J, Cha S, Aiken AH, Han ET, Crane JC, Stainsby JA, Wright GA, Dillon WP, Nelson SJ. Quantitative apparent diffusion coefficients and $\mathrm{T} 2$ relaxation times in characterizing contrast enhancing brain tumors and regions of peritumoral edema. J Magn Reson Imaging
2005;21:701-8.

12. Wen PY, Macdonald DR, Reardon DA, Cloughesy TF, Sorensen AG, Galanis E, Degroot J, Wick W, Gilbert MR, Lassman AB, Tsien C, Mikkelsen T, Wong ET, Chamberlain MC, Stupp R, Lamborn KR, Vogelbaum MA, van den Bent MJ, Chang SM. Updated response assessment criteria for high-grade gliomas: response assessment in neuro-oncology working group. J Clin Oncol 2010;28:1963-72.

13. Ellingson BM, Cloughesy TF, Lai A, Nghiemphu PL, Lalezari S, Zaw T, Motevalibashinaeini K, Mischel PS, Pope WB. Quantification of edema reduction using differential quantitative T2 (DQT2) relaxometry mapping in recurrent glioblastoma treated with bevacizumab. J Neurooncol 2012;106:111-9.

14. Hattingen E, Jurcoane A, Daneshvar K, Pilatus U, Mittelbronn M, Steinbach JP, Bähr O. Quantitative T2 mapping of recurrent glioblastoma under bevacizumab improves monitoring for non-enhancing tumor progression and predicts overall survival. Neuro Oncol 2013;15:1395-404.

15. Kern M, Auer TA, Picht T, Misch M, Wiener E. T2 mapping of molecular subtypes of WHO grade II/III gliomas. BMC Neurol 2020;20:8.

16. Cohen JF, Korevaar DA, Altman DG, Bruns DE, Gatsonis CA, Hooft L, Irwig L, Levine D, Reitsma JB, de Vet HC, Bossuyt PM. STARD 2015 guidelines for reporting diagnostic accuracy studies: explanation and elaboration. BMJ Open 2016;6:e012799.

17. Nabors LB, Portnow J, Ammirati M, Baehring J, Brem H, Butowski N, Fenstermaker RA, Forsyth P, Hattangadi-Gluth J, Holdhoff M, Howard S, Junck L, Kaley T, Kumthekar P, Loeffler JS, Moots PL, Mrugala MM, Nagpal S, Pandey M, Parney I, Peters K, Puduvalli VK, Ragsdale J, Rockhill J, Rogers L, Rusthoven C, Shonka N, Shrieve DC, Sills AK, Swinnen LJ, Tsien C, Weiss S, Wen PY, Willmarth N, Bergman MA, Engh A. NCCN Guidelines Insights: Central Nervous System Cancers, Version 1.2017. J Natl Compr Canc Netw 2017;15:1331-45.

18. Johnson DR, Guerin JB, Giannini C, Morris JM, Eckel LJ, Kaufmann TJ. 2016 Updates to the WHO Brain Tumor Classification System: What the Radiologist Needs to Know. Radiographics 2017;37:2164-80.

19. Cuccarini V, Erbetta A, Farinotti M, Cuppini L, Ghielmetti F, Pollo B, Di Meco F, Grisoli M, Filippini G, Finocchiaro G, Bruzzone MG, Eoli M. Advanced MRI may complement histological diagnosis of lower grade 
gliomas and help in predicting survival. Journal of neurooncology 2016;126:279-88.

20. Jeong J, Wang L, Ji B, Lei Y, Ali A, Liu T, Curran WJ, Mao H, Yang X. Machine-learning based classification of glioblastoma using delta-radiomic features derived from dynamic susceptibility contrast enhanced magnetic resonance images: Introduction. Quant Imaging Med Surg 2019;9:1201-13.

Cite this article as: Gu W, Fang S, Hou X, Ma D, Li S. Exploring diagnostic performance of $\mathrm{T} 2$ mapping in diffuse glioma grading. Quant Imaging Med Surg 2021;11(7):2943-2954. doi: 10.21037/qims-20-916 